\title{
Relationship of neck disability index, shoulder pain and disability index, and visual analogue scale in individuals with chronic neck pain
}

\author{
Sujin Hwang ${ }^{a}$, Mee-Hyang Mun \\ aDepartment of Physical Therapy, Division of Health Science, Baekseok University, Cheonan, Republic of Korea \\ ${ }^{\mathrm{b}}$ Physical Therapy Team, Yonsei University College of Medicine, Seoul, Republic of Korea
}

Objective: Several muscles surrounding neck are vital not only for neck motion, but for upper extremities motions as well. Neck pain would affect neck and shoulder disability. The Neck Disability Index (NDI), Shoulder Pain and Disability Index (SPADI), and Visual Analogue Scale (VAS) are increasingly used to evaluate treatment effectiveness after chronic neck pain. The purpose of this study was to analyze the correlation of neck pain, shoulder pain, and quality of life in patients with chronic neck pain.

Design: Cross-sectional study.

Methods: Forty-three patients with neck pain participated in this study. Participants were instructed to place a mark on each item of three clinical measures that best represented their experience of his/her neck and shoulder problem over the last week. The outcome measures of the study were NDI, SPADI, and VAS for neck and shoulder pain and disability.

Results: The NDI was significantly correlated with the VAS-Neck, SPADI-Pain, SPADI-Disability, SPADI-Total, and VAS-Shoulder $(p<0.05)$. The VAS-Neck was significantly correlated with the VAS-Neck, SPADI-Pain, SPADI-Disability, SPADITotal, and VAS-Shoulder $(p<0.05)$. The SPADI-Total was significantly correlated with the NDI, VAS-Neck, SPADI-Pain, SPADIDisability, and VAS-Shoulder $(p<0.05)$. The SPADI-Pain was significantly correlated with the NDI, VAS-Neck, SPADIDisability, SPADI-Total, and VAS-Shoulder $(p<0.05)$. The SPADI-Disability was significantly correlated with the NDI, VASNeck, SPADI-Total, SPADI-Pain, and VAS-Shoulder $(p<0.05)$. The VAS-Shoulder was significantly correlated with the NDI, VAS-Neck, SPADI-Pain, SPADI-Disability, and SPADI-Total $(p<0.05)$.

Conclusions: Neck disability in patients with chronic neck pain should be considered with disability and pain of the shoulder.

Key Words: Neck disability, Pain, Shoulder disability

\section{Introduction}

Musculoskeletal problems generally range from discomfort, minor aches and pains, to more serious medical conditions such as musculoskeletal disorders (MSD). Approximately $80 \%$ of the population will suffer from MSDs during their daily activities and at least 100 million people reported chronic musculoskeletal pain. The MSDs affect the body's muscles, joints, tendons, nerves, and resulting in limited functional activities in the home and the com- munity [1,2].

One of most frequently occurrencing Would be neck pain. The neck is included in the vertebral column, as its position influences the position of the spine. Because the neck provides stability and mobility for hand function and other upper extremity activities, the neck pain is applied for the vertebral column serves as an intermediary to transfer and absorb forces between the upper and lower extremities $[2,3]$.

In recent years, numerous studies have shown that the prevalence of neck pain and lower back pain in adolescents

Received: 2 October, 2013 Revised: 30 November, 2013 Accepted: 15 December, 2013

Corresponding author: Mee-Hyang Mun

Physical Therapy Team, Yonsei University College of Medicine, 50 Yonsei-ro, Seodaemun-gu, Seoul 120-749, Republic of Korea Tel: 82-2-2228-3780 Fax: 82-2-364-0509 E-mail: mmh1010@yuhs.ac

(c) This is an Open-Access article distributed under the terms of the Creative Commons Attribution Non-Commercial License (http://creativecommons.org/licens es/by-nc/3.0) which permits unrestricted non-commercial use, distribution, and reproduction in any medium, provided the original work is properly cited.

Copyright $@ 2013$ Korean Academy of Physical Therapy Rehabilitation Science 
is increasing [4-6]. They reported that the occurrence of neck pain and lower back pain is related to many factors, including depression, physical activity, and life style. Although previous studies have been designed significantly and specifically to assess neck pain and lower back pain, there is limited research concerning the correlation between neck pain and shoulder pain. Patient-reported outcome measures or health-related quality of life surveys are increasingly becoming the standard measure for treatment effectiveness in rehabilitation centers [7]. The purpose of this study was to investigate the correlation between neck pain, shoulder pain, and quality of life using the Neck Disability Index (NDI), the Shoulder Pain and Disability Index (SPADI), and the Visual Analogue Scale (VAS).

\section{Methods}

Forty-three patients with chronic neck pain participated in this study to investigate the correlation between neck and shoulder pain and disability. Volunteer participants were recruited using a brochure describing the study purpose, inclusion criteria and information about the researchers. All subjects provided an informed consent before participation. Data collection was performed in the outpatient physical therapy of adult rehabilitation units (Daejeon and Seoul, Korea). General characteristics of participants are shown in Table 1. The following inclusion criteria were used: a medically confirmed diagnosis of neck pain for six months or more since the onset of pain and absence of any cognitive impairment. Patients were excluded from the study if they had a history of any other orthopedic or neurologic disorders or any cognitive deficits that precluded support with procedures of this study.

\section{Clinical measures}

\section{Neck disability index}

The NDI is a patient-reported outcome measure, and is a 10 -item self-administered questionnaire measuring dis-

Table 1. Common characteristic of the participants in this study

$(\mathrm{N}=43)$

\begin{tabular}{lc}
\hline \multicolumn{1}{c}{ Variable } & Data \\
\hline Sex (male/female) & $10 / 33$ \\
Age (yr) & $48.81(10.00)$ \\
Post-duration & $16.84(13.54)$ \\
\hline
\end{tabular}

Values are presentd as number only or mean (SD). ability in patients with neck pain. Each item is scored from 0 to 5 with a maximum score of 50 . The higher the score, the greater the disability. The test-retest correlations that have been reported to range from 0.90 to 0.93 , with Cronbach alpha ranging from 0.74 to 0.93 . Correlations of the NDI with measures of global change range from 0.30 to $0.76[8,9]$.

\section{Shoulder pain and disability index}

The SPADI was developed to measure current shoulder pain and disability with musculoskeletal, neurogenic, or undetermined conditions [10]. The SPADI contains 13 items that assess two domains; pain subscale (5-item) and disability subscale (8-item). This study used the numerical rating scale version of the SPADI. It is an 11 item-ordinal scale from 0 (no pain or no disability) to 10 (worst pain imaginable or so difficult it requires help). Each subscale is summed and transformed to a score out of 100. A mean is taken of the two subscales to give a total score out of 130 with a higher score indicating greater impairment or disability. Reliability coefficients of Intraclass correlation coefficient $\geq 0.89$ in a variety of patient populations. The SPADI demonstrates good construct validity, correlating well with other region-specific shoulder questionnaires $[11,12]$.

\section{Visual analogue scale}

The VAS is a psychometric response scale which can be used in questionnaires for subjective characteristics or attitudes that cannot be directly measured. The scale is most commonly anchored by 'no pain ( 0$)$ ' and 'worst imaginable pain (10)'. The starting point of ' 0 ', indicates no pain and the ending point of ' 10 ', indicates worst pain. Test-retest reliability and validity has been shown to be good in patients with chronic pain conditions $[13,14]$.

\section{Procedures}

Clinical measures were assessed in a quiet and well-organized therapy room and participants were given the standard verbal instructions of the NDI, SPADI, and VAS. Two examiners except authors involved in this study. The examiner gave a detailed explanation of the purpose and testing methods of three clinical tools before responding to clinical measures. Participants were instructed to place a mark on each item of three clinical measures that best represented their experience of his/her neck and shoulder problem over the last week. Clinical measures were taken according to consensus-based standards for the selection of health status measurement instruments standards. 


\section{Data analysis}

The dependent variables of this study were scores on the NDI, VAS-Neck, SPADI-Pain, SPADI-Shoulder, and SPADITotal, and VAS-Shoulder. Descriptive analysis was used to describe the common characteristics including sex, age, and post-neck pain duration, and the scores of clinical measures such as NDI, VAS-Neck, SPADI-Pain, SPADI-Shoulder, and SPADI-Total, and VAS-Shoulder. IBM SPSS Statistics version 19.0 (IBM Co., Amonk, NY, USA) was used for all statistical analysis. Pearson's correlations were used to quantify the bivariate associations between the NDI, VASNeck, SPADI-Pain, SPADI-Shoulder, SPADI-Total, and VASShoulder. $p<0.05$ was defined as significantly different.

\section{Results}

The mean scores for the NDI, VAS-Neck, SPADI-Pain, SPADI-Shoulder, SPADI-Total, and VAS-Shoulder are shown in Table 2. The relationship between NDI, VASNeck, SPADI-Pain, SPADI-Disability, SPADI-Total, and

Table 2. Mean and standard deviation of the clinical measures

$(\mathrm{N}=43)$

\begin{tabular}{lrc}
\hline Clinical measure & Mean & Standard deviation \\
\hline NDI & 20.81 & 11.72 \\
VAS-N & 4.65 & 2.51 \\
SPADI-Pain & 24.88 & 11.39 \\
SPADI-Disability & 27.05 & 11.81 \\
SPADI-Total & 51.93 & 26.29 \\
VAS-Shoulder & 5.30 & 1.95 \\
\hline
\end{tabular}

NDI: Neck Disability Index, VAS-N: Visual Analogue Scale for Neck, SPADI-Pain: Shoulder Pain and Disability Index-Pain, SPADIDisability: Shoulder Pain and Disability Index-Disability, SPADITotal: Shoulder Pain and Disability Index-Total, VAS-Shoulder: Visual Analogue Scale for Shoulder.
VAS-Shoulder are shown in Table 3. The NDI was significantly correlated with the VAS-Neck $(\mathrm{r}=0.691)$, SPADIPain $(\mathrm{r}=0.817)$, SPADI-Disability ( $\mathrm{r}=0.720)$, SPADI-Total $(\mathrm{r}=0.787)$, and VAS-Shoulder $(\mathrm{r}=0.611)$. The VAS-Neck was significantly correlated with the VAS-Neck $(\mathrm{r}=0.691)$, SPADI-Pain ( $\mathrm{r}=0.435)$, SPADI-Disability ( $\mathrm{r}=0.422)$, SPADITotal $(\mathrm{r}=0.442)$, and VAS-Shoulder $(\mathrm{r}=0.740)$. The SPADITotal was significantly correlated with the NDI $(\mathrm{r}=0.787)$, VAS-Neck $(\mathrm{r}=0.442)$, SPADI-Pain $(\mathrm{r}=0.954)$, SPADI-Disability ( $\mathrm{r}=0.976$ ), and VAS-Shoulder $(\mathrm{r}=0.554)$. The SPADIPain was significantly correlated with the NDI $(\mathrm{r}=0.817)$, VAS-Neck ( $\mathrm{r}=0.435)$, SPADI-Disability $(\mathrm{r}=0.865)$, SPADITotal $(\mathrm{r}=0.954)$, and VAS-Shoulder $(\mathrm{r}=0.503)$. The SPADIDisability was significantly correlated with the NDI ( $\mathrm{r}=$ $0.720)$, VAS-Neck ( $\mathrm{r}=0.422)$, SPADI-Total $(\mathrm{r}=0.976)$, SPADIPain $(\mathrm{r}=0.865)$, and VAS-Shoulder $(\mathrm{r}=0.503)$. The VASShoulder was significantly correlated with the NDI $(r=$ $0.611)$, VAS-Neck ( $\mathrm{r}=0.740)$, SPADI-Pain ( $\mathrm{r}=0.580)$, SPADIDisability $(\mathrm{r}=0.503)$, and SPADI-Total $(\mathrm{r}=0.554)$.

\section{Discussion}

The purpose of this study was to investigate the correlation between the neck pain and disability, and shoulder pain and disability in patients with chronic neck pain. The results of this study revealed several significant findings. First the score of NDI showed good correlation with VAS-Neck score, SPADI-Pain score, SAPDI-Disability score, SPADITotal score, and VAS-Shoulder score. Therefore, the results showed that neck disability showed good correlation with neck pain, shoulder pain, and shoulder disability. Second, total SPADI score showed a good correlation with NDI score, VAS-Neck score, SPADI-Pain score, SAPDI-Disability score, and VAS-Shoulder score. The results showed that shoulder pain and disability are a good relationship with

Table 3. Correlations among the neck and shoulder pain

$(\mathrm{N}=43)$

\begin{tabular}{lcccccc}
\hline & NDI & VAS-Neck & SPADI-Total & SPADI-Pain & SPADI-Disability & VAS-Shoulder \\
\hline NDI & 1 & $0.691^{*}$ & $0.787^{*}$ & $0.817^{*}$ & $0.720^{*}$ & $0.611^{*}$ \\
VAS-Neck & $0.691^{*}$ & 1 & $0.442^{*}$ & $0.435^{*}$ & $0.422^{*}$ & $0.740^{*}$ \\
SPADI-Total & $0.787^{*}$ & $0.442^{*}$ & 1 & $0.953^{*}$ & $0.976^{*}$ & $0.554^{*}$ \\
SPADI-Pain & $0.817^{*}$ & $0.435^{*}$ & $0.954^{*}$ & 1 & $0.865^{*}$ & $0.580^{*}$ \\
SPADI-Disability & $0.720^{*}$ & $0.422^{*}$ & $0.976^{*}$ & $0.865^{*}$ & 1 & $0.503^{*}$ \\
VAS-Shoulder & $0.611^{*}$ & $0.740^{*}$ & $0.554^{*}$ & $0.580^{*}$ & $0.503^{*}$ & 1 \\
\hline
\end{tabular}

NDI: Neck Disability Index, VAS-Neck: Visual Analogue Scale for Neck, SPADI-Pain: Shoulder Pain and Disability Index-Pain, SPADI-Disability: Shoulder Pain and Disability Index-Disability, SPADI-Total: Shoulder Pain and Disability Index-Total, VAS-Shoulder: Visual Analogue Scale for Shoulder. ${ }^{*} p<0.01$. 
neck pain and disability.

Many researchers have been interpreted that neck pain would affect lower back conditions. Royuela et al. [15] investigated to develop models that predict the improvement of spinal pain and disability in patients with chronic neck or lower back pain undergoing conservative treatment They reported that neck and lower back pain in routine practice can be used to develop models that estimate the probably of improvement for each individual patient undergoing a specific form of treatment. Pedersen et al. [16] evaluated the long-term adherence and effects of a workplace strength training intervention on back, neck and upper extremity pain among laboratory technicians. They reported that specific strength training at the workplace can lead to significant long-term reductions in spinal and upper extremity pain. However, there is insufficient evidence to examine the correlations between neck disability, neck pain, shoulder disability, and shoulder pain. The purposes of this study was to examine the correlation between neck and shoulder function in patients with chronic neck pain. The results showed that neck pain and disability showed good relationship with shoulder pain and disability in this study. The results of this study suggested that neck pain and disability is a matter of the way in which each individual with chronic neck pain interprets the effect of the chronic neck pain on his or her functional activities using the shoulder complex. Neck disability would be as difficulty doing activities using shoulder complex in any domain of life. Currently, work-related neck and shoulder pain is an ongoing and increasing problem among office workers as well as high-school students. Neck and shoulder pain are caused by prolonged low-level static exertions as well as high visual, postural, and cognitive stress conditions. We conclude that future studies showed investigate the effect of decreasing neck pain on shoulder disability.

The study showed some limitations. Some participants had only mild functional complaints, possibly explaining he non-significant changes in disability. The study design in which subjects served as their own control may have some limitations. Further studies can be used to identify the clinical course of neck and shoulder pain and using its prognostic factors to assist in designing scales and prevention programs. The further study can be used to investigate whether treatments for neck pain and disability will affect improvement of shoulder pain and disability or not.

\section{References}

1. Canivet C, Ostergren PO, Choi B, Nilsson P, af Sillén U,
Moghadassi M, et al. Sleeping problems as a risk factor for subsequent musculoskeletal pain and the role of job strain: results from a one-year follow-up of the Malmö Shoulder Neck Study Cohort. Int J Behav Med 2008;15:254-62.

2. Barr KP. Review of upper and lower extremity musculoskeletal pain problems. Phys Med Rehabil Clin N Am 2007;18:747-60, vi-vii.

3. Houglum PA, Bertoti D, Brunnstrom S. Brunnstrom's clinical kinesiology. 6th ed. Philadelphia: F.A. Davis; 2012.

4. Hohmann C, Ullrich I, Lauche R, Choi KE, Lüdtke R, Rolke R, et al. The benefit of a mechanical needle stimulation pad in patients with chronic neck and lower back pain: two randomized controlled pilot studies. Evid Based Complement Alternat Med 2012;2012:753583.

5. Maigne JY, Cornelis $P$, Chatellier G. Lower back pain and neck pain: is it possible to identify the painful side by palpation only? Ann Phys Rehabil Med 2012;55:103-11.

6. Williams NH, Wilkinson C, Russell IT. Extending the Aberdeen Back Pain Scale to include the whole spine: a set of outcome measures for the neck, upper and lower back. Pain 2001;94:26174.

7. O'Sullivan SB, Schmitz TJ, Fulk GD. Physical rehabilitation. 6th ed. Philadelphia: F.A. Davis; 2014.

8. Vernon H, Mior S. The Neck Disability Index: a study of reliability and validity. J Manipulative Physiol Ther 1991;14:40915.

9. Vernon H. The Neck Disability Index: state-of-the-art, 19912008. J Manipulative Physiol Ther 2008;31:491-502.

10. Roach KE, Budiman-Mak E, Songsiridej N, Lertratanakul Y. Development of a shoulder pain and disability index. Arthritis Care Res 1991;4:143-9.

11. Angst F, Goldhahn J, Pap G, Mannion AF, Roach KE, Siebertz D, et al. Cross-cultural adaptation, reliability and validity of the German Shoulder Pain and Disability Index (SPADI). Rheumatology (Oxford) 2007;46:87-92.

12. Roddey TS, Olson SL, Cook KF, Gartsman GM, Hanten W. Comparison of the University of California-Los Angeles Shoulder Scale and the Simple Shoulder Test with the shoulder pain and disability index: single-administration reliability and validity. Phys Ther 2000;80:759-68.

13. Hielm-Björkman AK, Kapatkin AS, Rita HJ. Reliability and validity of a visual analogue scale used by owners to measure chronic pain attributable to osteoarthritis in their dogs. Am J Vet Res 2011;72:601-7.

14. Boonstra AM, Schiphorst Preuper HR, Reneman MF, Posthumus JB, Stewart RE. Reliability and validity of the visual analogue scale for disability in patients with chronic musculoskeletal pain. Int J Rehabil Res 2008;31:165-9.

15. Royuela A, Kovacs FM, Campillo C, Casamitjana M, Muriel A, Abraira V. Predicting outcomes of neuroreflexotherapy in patients with subacute or chronic neck or low back pain. Spine J 2013. [Epub ahead of print]

16. Pedersen MT, Andersen CH, Zebis MK, Sjøgaard G, Andersen LL. Implementation of specific strength training among industrial laboratory technicians: long-term effects on back, neck and upper extremity pain. BMC Musculoskelet Disord 2013;14: 287. 